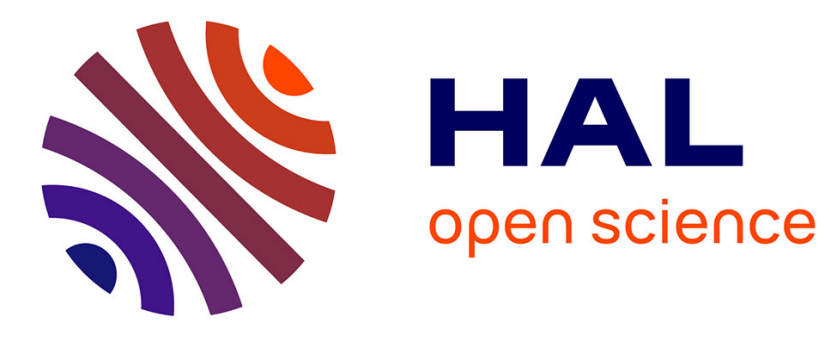

\title{
Automatic dendrite spines detection from X-ray tomography volumes
}

Xavier Descombes, Grégoire Malandain, Caroline Fonta, Lazlo Negyessy, Rajmund Mosko

\section{- To cite this version:}

Xavier Descombes, Grégoire Malandain, Caroline Fonta, Lazlo Negyessy, Rajmund Mosko. Automatic dendrite spines detection from X-ray tomography volumes. ISBI - International Symposium on Biomedical Imaging, Apr 2013, San fancisco, United States. 10.1109/ISBI.2013.6556505 . hal00793573

\section{HAL Id: hal-00793573 \\ https://hal.inria.fr/hal-00793573}

Submitted on 22 Feb 2013

HAL is a multi-disciplinary open access archive for the deposit and dissemination of scientific research documents, whether they are published or not. The documents may come from teaching and research institutions in France or abroad, or from public or private research centers.
L'archive ouverte pluridisciplinaire HAL, est destinée au dépôt et à la diffusion de documents scientifiques de niveau recherche, publiés ou non, émanant des établissements d'enseignement et de recherche français ou étrangers, des laboratoires publics ou privés. 


\title{
AUTOMATIC DENDRITE SPINES DETECTION FROM X-RAY TOMOGRAPHY VOLUMES
}

\author{
Xavier Descombes ${ }^{1}$, Grégoire Malandain ${ }^{1}$, Caroline Fonta ${ }^{2}$, Lázló Négyessy ${ }^{3}$, Rajmund Mosko ${ }^{4}$ \\ ${ }^{1}$ Morpheme, INRIA/I3S/ iBV, Sophia Antipolis France \\ ${ }^{2}$ CerCo, UMR5549, CNRS/UPS, Toulouse France \\ ${ }^{3}$ WRCP, Hungarian Academy of Science, Budapest, Hungary \\ ${ }^{4}$ SLS, PSI, Villigen, Switzerland
}

\begin{abstract}
We consider the problem of dendritic spine detection from $\mathrm{X}$-ray micro-tomographic volumes that allow huge volume of tissue visualization. To compensate for the noise in data that induces false positives in the spine detection process, we first segment the dendrites. This segmentation is obtained by computing the medial axis and approximating the results by segments obtained with a 3D Hough transform. Dendrites are then reconstructed and a spine mask is obtained using the typical diameter of dendrites and distance between spine and dendrites. A point process is then optimized on this mask, thus providing the spine detection.
\end{abstract}

Index Terms - Dendritic spine, X-Ray micro-tomography, 3D Hough transform, Point Process

\section{INTRODUCTION}

Dendritic spines are small bulbous structures connected to the stem dendrite through a tiny neck. They have a huge biological importance as they represent the majority of the synaptic connections of the cerebral cortex providing the backbone of the cortical connectome [1]. Studying their density and repartition along dendrites provide essential information to explain neural network functioning, and pathology of diverse disorders $[2,3,4,5]$. In addition, their repartition according to a morphologic type (mushroom, stubby or thin) provides more detailed quantative biomarkers.

The first step to study spine repartition consists in detecting and segmenting the dendritic spines in neurons images. In the literature, imaging protocols generally rely on fluorescence microscopy techniques, either confocal scanning microscopy [6], 2-photons scanning microscopy [7, 8], or electron microscopic reconstruction. Such protocols provide contrasted images at high resolution that facilitate the detection process. However, the obtained images are usually anisotropic, with a slice thickness that may be much larger than the pixel size. As a consequence, some spines may be missed. In addition, the imaged field of view of the scanning microscopy technique is quite small (typically one hundred of
$512 \times 512$ slices with a pixel size of about $0.05 \mu \mathrm{m}$ ): a larger number of samples is required for statistical analysis.

In this paper, we consider an alternative imaging protocol, namely the X-rays micro-tomography (XRMT). It overcomes the two main drawbacks of the scanning microscopy protocols, since the obtained 3D images are isotropic (the voxel size is identical along the 3 dimensions), and it allows to image larger samples (typically the obtained images are of size $1000^{3}$ with a voxel size of $0.308 \mu \mathrm{m}^{3}$ ). Moreover, in contrast to scanning microscopy, no occlusion or masking by overlapping structures occur in the deeper slices (structures closer to the objective lens occlude deeper ones beneath).

One advantage of fluorescence microscopy is due to the potential use of dedicated labels, which are cellular specific. However, they require either intracellular staining or use of transgenic animals. Moreover, photo-bleaching is clearly a limitation to such staining. Our staining technique (Golgi [9]) is more versatile, and is clearly sufficient to describe the fundamental brain circuits with a high specificity (Ramon y Cajal describes the different morphologic spine types with such staining at the end of the 19th century).

XMRT also exhibits a higher level of noise than laser scanning microscopy. This implies that popular existing approaches (e.g. SpineLab [10] or Neuronstudio [6]) are likely to fail. Hence, robust algorithms are mandatory. Moreover, some of the existing methods requires user interaction, which is not compatible with the large volumes produced by XMRT. Therefore, a fully automated approach has to be designed.

Before defining a strategy to detect dendritic spines, let us notice some difficulties linked with the problem: (i) spine size is close to the data resolution, (ii) spine may appear not connected with the dendrite. Therefore we have to avoid (i) denoising (as proposed in [7]) that may remove some spine, (ii) basing the detection on the dendrite tree extraction (as proposed in [6]) that may omit some spines and (iii) detecting spines on the whole dataset that may introduce many false positives (as proposed in [7]). Besides, we expect to make the detection in 3D but not on the Maximum Intensity Projection as in [11]. To circumvent these difficulties we propose to use shape information and localization with respect to dendrites 
to detect spines. We first extract dendrites as tubular structures. These tubular features are used to define a spatial mask where spine may appear. A spine detector embedding shape information is finally applied on this mask.

We detail the method in section 2 and show some result in section 3 before concluding in section 4 .

\section{METHODS}

\subsection{Extracting dendrite medial axis}

First, the medial axis of the dendrites are detected thanks to a multi-scale Hessian-based method [12]. Basically, at each scale $\sigma$ the Hessian matrix (i.e. the matrix of the image second derivatives) is calculated by a convolution of the image $I$ with the second derivatives of a 3D Gaussian kernel of standard deviation $\sigma$. At the center of a tubular structure, the Hessian matrix exhibits two high eigenvalues, whose associated eigenvectors $\mathbf{v}_{1}$ and $\mathbf{v}_{2}$ are orthogonal to the direction of the tubular structure, and one low eigenvalue, whose associated eigenvector obviously gives the direction of the tubular structure. Once the likely direction of the dendrite is identified at point $M$, a function response is calculated that integrates the radial derivative along a circle of radius $\sigma$ :

$$
R_{\sigma}(M)=1 / 2 \pi \int_{0}^{2 \pi} \mathbf{v}(\theta) \cdot \nabla_{\sigma} I(M+\sigma \mathbf{v}(\theta)) d \theta
$$

with $\mathbf{v}(\theta)=\cos \theta \mathbf{v}_{1}+\sin \theta \mathbf{v}_{2}$.

The responses at each scale are then combined into a multiscale response, thanks to normalized $\gamma$-derivatives [13], and last the local extrema of this response, with respect to the directions $\mathbf{v}_{1}$ and $\mathbf{v}_{2}$ are extracted.

\subsection{Dendrite detection}

To denoise the obtained dendrite medial axis image, we consider a segment approximation by computing the 3D Hough transform. To parametrize lines in the 3D space, we consider a sphere including the data volume. Any line crossing the sphere is then parametrized by the four angles corresponding to the two intersection points between the line and the sphere. Any voxel $P$ above a threshold $T$, in the medial axis image will vote for the following points in the 4D Hough space (see figure 1):

$$
\begin{gathered}
\left\{\left(\theta_{1}, \phi_{1}, \theta_{2}, \phi_{2}\right), \quad \theta_{1}, \theta_{2} \in[-\pi, \pi], \phi_{1}, \phi_{2} \in\left[0, \frac{\pi}{2}\right],\right. \\
\left.P S_{1} \| S_{1} S_{2},\right\},
\end{gathered}
$$

where $S_{1}$ (resp, $S_{2}$ ) is the point on the sphere with angles $\left(\theta_{1}, \phi_{1}\right)$ (resp. $\left.\left(\theta_{2}, \phi_{2}\right)\right)$ The candidate lines are obtained by extracting the local maxima in the Hough space. Finally, the dendrites are approximated from the candidate lines by considering the segments constituted of voxels whose gray value

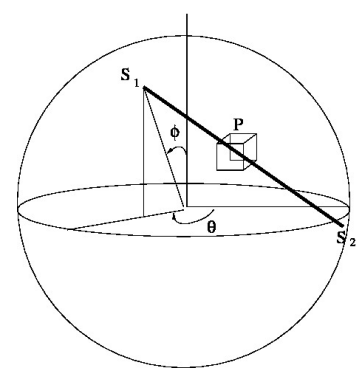

Fig. 1. The voting mechanism for the 3D Hough transform

is higher than the threshold $T$ and having a minimal length $l$. Once the segments describing the dendrite axis have been obtained, we reconstruct the dendrites with a conditional dilation $M_{D}=\left(S_{D} \oplus K_{1}\right) \cap Y_{T}$, where $\oplus$ is the Minkowsky addition and $Y_{T}$ is the data thresholded at $T$. As dendrites are mainly vertically oriented, we consider a disk in the XY plane for the structuring element $K_{1}$, the size of which is defined by the data resolution and the typical dendrites diameter (three voxels in our case as the typical dendrite diameter is $1 \mu m$ [14]).

\subsection{Spine detection}

Once we have detected dendrites we define a mask where spines may lie. This mask is obtained using a dilation of the dendrite mask as follows:

$$
M_{S}=M_{D} \oplus K_{2}-M_{D}
$$

where $M_{D}$ is the dendrite mask, $\oplus$ the Minkowsky addition. The size of the structuring element $K_{2}$ is defined by the resolution and the maximum distance between a spine and its dendrite (again, a XY disk of size three voxels is used as a structuring element corresponding to the typical size of spine stalk [14]). The spine mask $M_{S}$ allows a reduction of the computation time in the detection process but also avoids false positives due to irregularities on dendrites.

To detect spines we consider a point process in $M_{S}$. A configuration of points is denoted $x=\left\{x_{1}, \ldots, x_{n}\right\}, x_{i} \in$ $M_{S}$. This probabilistic framework embeds geometric constraints through the definition of a data term and structural constraints through the definition of a prior. It has been successfully applied for multiple objects detection problems such as trees, flamingos or cells detection. We define the point process by an unormalized density $h$ with respect to the Poisson measure [15] as follows:

$$
h(x)=f(x) g(x \mid y)
$$

where $f(x)$ is the prior and $g(x \mid y)$ the data term depending on data $y$. The prior is a hard core process:

$$
f(x)=\prod_{(i, j), i \neq j} \delta\left(d\left(x_{i}, x_{j}\right)>D\right),
$$


where $\delta(a)=1$ if $a$ is true and 0 otherwise. Therefore the prior states that the minimal distance between two spines must be greater than $D$ ( $D=3$ voxels in our case). To compute the data term we consider that a spine is included in a $3 \times 3 \times 3$ cube (the typical spine bulb size being $1 \mu \mathrm{m}$ in mammals [14]) and consider the contrast between this cube and its neighborhood by computing the Bhattacharyya distance:

$b\left(x_{i}\right)=\left[\frac{1}{4} \frac{\left(\mu_{\text {in }}-\mu_{\text {out }}\right)^{2}}{\sigma_{\text {in }}^{2}+\sigma_{\text {out }}^{2}}+\frac{1}{2} \log \left(\frac{\sigma_{\text {in }}^{2}+\sigma_{\text {out }}^{2}}{2 \sqrt{\sigma_{\text {in }}^{2} \sigma_{\text {out }}^{2}}}\right)\right] \delta\left(\frac{\mu_{\text {in }}}{\mu_{\text {out }}}>1\right)$,

where $\mu_{\text {in }}$ and $\sigma_{\text {in }}^{2}$ (resp. $\mu_{\text {out }}$ and $\sigma_{\text {out }}^{2}$ ) are the mean and variance of the set $\left\{y_{s}: d(i, s)<2\right\}$ (resp. $\left\{x_{s}: 2 \leq\right.$ $d(i, s)<3\})$. Finally, the data is written as follows:

$$
g(x \mid y)=\exp \left[-\sum_{i} u\left(x_{i}\right)\right]
$$

where:

$$
u\left(x_{i}\right)= \begin{cases}1-\frac{b\left(x_{i}\right)}{b_{0}} & \text { if } b\left(x_{i}\right)<b_{o} \\ \exp \frac{-\left(b\left(x_{i}\right)-b_{o}\right)}{3 b_{0}} & \text { otherwise }\end{cases}
$$

$b_{0}$ is a contrast parameter that defines spine candidates when the corresponding Bhattacharyya distance is greater than $b_{0}$.

To obtain the optimal solution with respect to $h($.$) , we$ consider a fast approximation of the Multiple Birth and Death algorithm [16]:

Birth: Consider as candidates the set $C$ of all points in $M_{S}$

Sort: Sort the candidates $x_{i} \in C$ with respect to $u\left(x_{i}\right)$ in the decreasing order (the worst first), to obtained $x=$ $\left\{x_{1}, \ldots, x_{n}\right\}$

Death: For $i=1$ to $n$, if $h(x)<h\left(x /\left\{x_{i}\right\}\right)$ remove $x_{i}$ from $x$

\section{RESULTS}

To exemplify the proposed approach, a subvolume $(220 \times$ $180 \times 100)$ has been extracted from a XRMT volume that is given on figure 2. As expected, the spines appear as small objects, whose size is close to the image resolution, along the tubular structures representing dendrites (see figure 3). Using the localization information to detect spine is essential to prevent false positives due to noise or to the deviation of dendrites from a cylinder model (see figure 4 for a potential false positive avoided thanks to its distance with respect to dendrites). The parameters linked with the geometry (size of the mask and structuring elements) are automatically fixed considering the image resolution and the dendrite spine size referred in the litterature [14]. The remaining parameters (threshold in the 3D Hough transform and in the detection step) are data dependant as they are linked with the image contrast. Next step will consists in estimating these parameters from the image histogram.
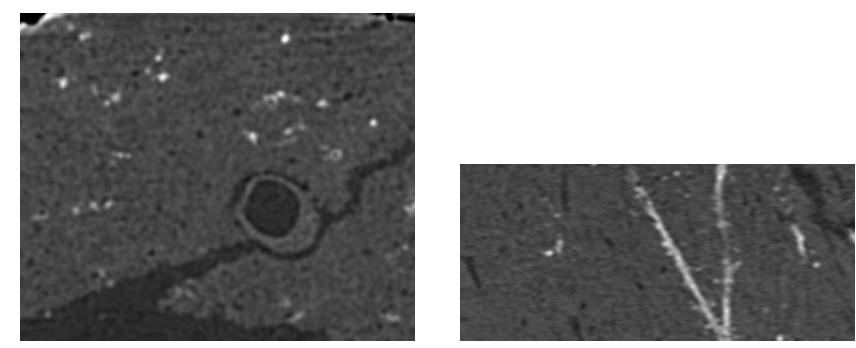

Fig. 2. Original XMRT data. Left: axial slice $(69 \mu \mathrm{m} \times 57 \mu m)$ ; right: sagittal slice $(69 \mu m \times 31 \mu m)$.
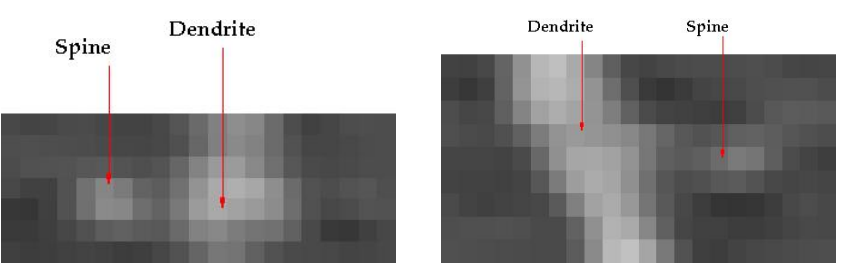

Fig. 3. Examples of spines. Left: constrasted spine; right: poorly contrasted spine.

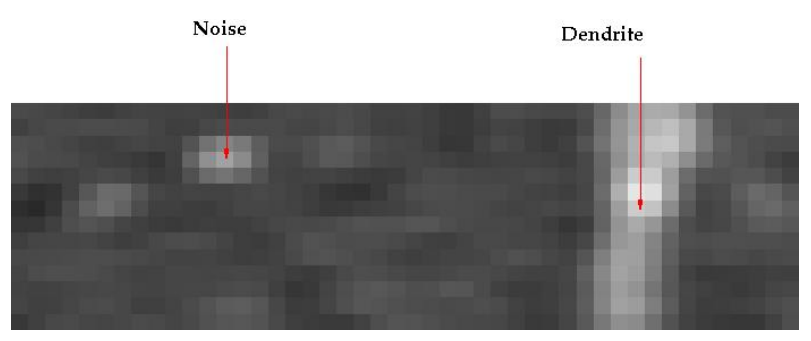

Fig. 4. Noise similar to spine but too far from dendrite.

Figure 5 shows the detected dendrite medial axis and the spine mask derived from the dendrite detection. The obtained spine detection is shown on figure 6 . The obtained results are promising and correspond to a visual inspection of the data. We are currently performing a manual detection by several experts to provide a quantitative evaluation of these results.

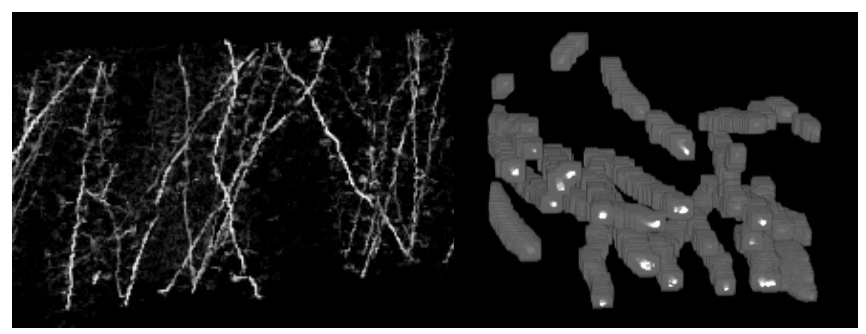

Fig. 5. Definition of a spine mask. Left: medial axis (perspective view from front side); right: dendrite in white and spine mask in gray (perspective view from top). 


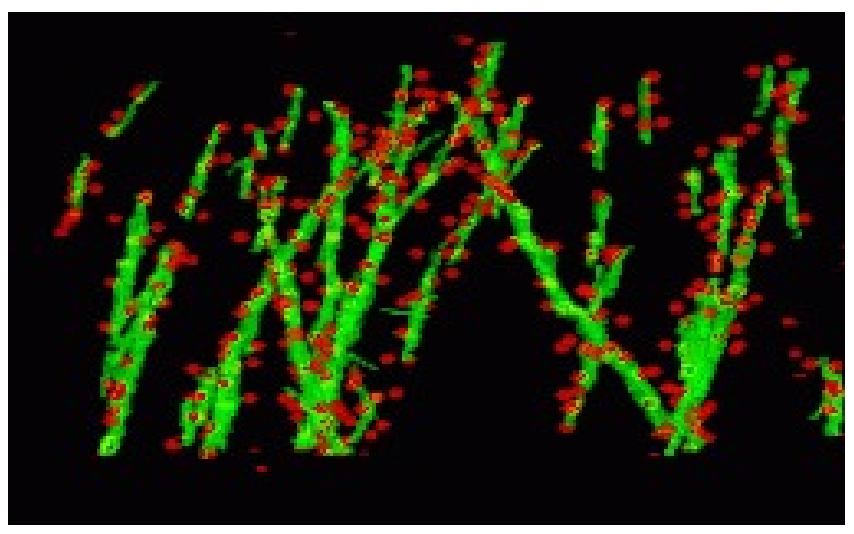

Fig. 6. Dendrites (in green) and spines (in red)

\section{CONCLUSION}

We have proposed an automated algorithm for detecting dendritic spines from XRMT data. XRMT data allows imaging a large volume of tissue, and therefore a higher number of spines than laser scanning microscopy. We have shown that despite the lower image quality compared to microscopic data, we were able to extract dendritic spines. The main idea of the proposed approach is to define a mask for performing the spine detection without facing the false positive problem. We therefore first extract the dendrites themselves and then compute the spine mask based on prior knowledge on their localization with respect to dendrites. Results are visually promising, and a forthcoming validation study will allow to better assess the quality of the detection by providing a quantitative evaluation. In a next step, we will consider the problem of spine classication in the three classical classes: stubby, thin, mushroom.

\section{REFERENCES}

[1] R. Yuste, "Dendritic spines and distributed circuits," Neuron, vol. 71, no. 5, pp. 772-81, September 2011.

[2] J.C. Fiala, J. Spacek, and K.M. Harris, "Dendritic spine pathology: cause or consequence of neurological disorders?," Brain Res Brain Res Rev, vol. 39, no. 1, pp. 29-54, June 2002.

[3] S.A. Irwin, R. Galvez, and W.T. Greenough, "Dendritic spine structural anomalies in fragile-x mental retardation syndrome," Cereb Cortex, vol. 10, no. 10, pp. 103844, October 2000.

[4] C. Tackenberg, A. Ghori, and R. Brandt, "Thin, stubby or mushroom: spine pathology in alzheimer's disease," Curr Alzheimer Res, vol. 6, no. 3, pp. 261-8, June 2009.

[5] K.W. Lee, Y. Kim, A.M. Kim, K. Helmin, A.C. Nairn, and P. Greengard, "Cocaine-induced dendritic spine formation in $\mathrm{d} 1$ and $\mathrm{d} 2$ dopamine receptor-containing medium spiny neurons in nucleus accumbens," Proc Natl Acad Sci U S A, vol. 103, no. 9, pp. 3399-404, February 2006.

[6] A. Rodriguez, D.B. Ehlenberger, D.L. Dickstein, P.R. Hof, and S.L. Wearne, "Automated three-dimensional detection and shape classification of dendritic spines from fluorescence microscopy images," PLoS One, vol. 3, no. 4, 2008.

[7] J. Cheng, X. Zhou, E. Miller, R.M. Witt, J. Zhu, B.L. Sabatini, and S.T.C. Wong, "A novel computational approach for automatic dendrite spines detection in twophoton laser scan microscopy," J Neurosci Methods, vol. 165, no. 1, pp. 122-34, September 2007.

[8] Y. Zhang, X. Zhou, R.M. Witt, B.L. Sabatini, D. Adjeroh, and S.T.C. Wong, "Dendritic spine detection using curvilinear structure detector and lda classifier," Neuroimage, vol. 36, no. 2, pp. 346-60, June 2007.

[9] A. Fairen, A. Peters, and J. Saldanha, "A new procedure for examining golgi impregnated neurons by light and electron microscopy," J Neurocytol, vol. 6, no. 3, pp. 311-37, June 1977.

[10] D. Jungblut, A. Vlachos, G. Schuldt, N. Zahn, T. Deller, and G. Wittum, "Spinelab: tool for three-dimensional reconstruction of neuronal cell morphology," J Biomed Opt, vol. 17, no. 7, pp. 076007, July 2012.

[11] S.K. Choy, K. Chen, Y. Zhang, M. Baron, M.A. Teylan, Y. Kim, C.S. Tong, Z. Song, and S.T. Wong, "Multi scale and slice-based approach for automatic spine detection," in Proc. Eng. Med. Biol. Soc. IEEE, pp. 47654768.

[12] K. Krissian, G. Malandain, N. Ayache, R. Vaillant, and Y. Trousset, "Model-based detection of tubular structures in 3d images," Comp. Vision and Im. Understanding, vol. 80, no. 2, pp. 130-171, 2000.

[13] T. Lindeberg, Scale-Space Theory in Computer Vision, Kluwer Academic Publishers, Dordrecht, Netherlands, 1994.

[14] S. Jacobson, "Dimensions of the dendritic spine in the sensorimotor cortex of the rat, cat, squirrel monkey and man," J. Comp. Neur., vol. 128, pp. 49-58, 2004.

[15] X. Descombes, Ed., Stochastic geometry for image analysis, Wiley/Iste, 2011.

[16] X. Descombes, R. Minlos, and E. Zhizhina, "Object extraction using a stochastic birth-and-death dynamics in continuum," J. of Math. Imaging and Vision, vol. 33, no. 3, pp. 347-359, 2009. 